3. J. L. Walsh, Interpolation and approximation by rational functions in the complex domain, Amer. Math. Soc. Colloquium Publications, vol. 20, 1935.

4. - The location of critical points of analytic and harmonic functions, Amer. Math. Soc. Colloquium Publications, vol. 34, 1950.

5. - An interpolation problem for harmonic functions, Amer. J. Math. vol. 76 (1954) pp. 259-272. Also Determination d'une fonction analytique par ses valeurs données dans une infinité denombrable de points, Bull. Soc. Math. de Belgique (1955) pp. 52-70.

6. J. L. Walsh and Philip Davis, Interpolation and orthonormal systems, Journal d'Analyse Mathématique vol. 2 (1952) pp. 1-28.

HARVARD UNIVERSITY AND

Smith College

\title{
PARTIALLY BOUNDED CONTINUED FRACTIONS
}

\author{
H. S. WALL
}

For each complex number sequence $a, f(a)$ denotes the continued fraction

$$
\frac{1}{1}+\frac{a_{1}}{1}+\frac{a_{2}}{1}+\frac{a_{3}}{1}+\cdots
$$

The statement that $f(a)$ is partially bounded ${ }^{1}$ means that the sequence $a$ has a bounded infinite subsequence. If $f(a)$ is partially bounded, the series $\sum\left|b_{p}\right|$ diverges, where $b_{1}=1, a_{p}=1 / b_{p} b_{p+1}, p=1,2, \cdots,-\mathrm{a}$ necessary condition for convergence of $f(a)$.

Any continued fraction $f(a)$ such that $\sum\left|b_{p}\right|$ diverges is convergent provided its even and odd parts are absolutely convergent, ${ }^{2}$ i.e. provided the series $\sum\left|f_{2 p+2}-f_{2 p}\right|$ and $\sum\left|f_{2 p+1}-f_{2 p-1}\right|$ are convergent, where $\left\{f_{p}\right\}_{p=1}^{\infty}$ is the sequence of approximants. The simple convergence of the even and odd parts of $f(a)$, together with the divergence of $\sum\left|b_{p}\right|$, is not sufficient for the convergence of $f(a)$, (Theorem 3). However, the simple convergence of the even and odd parts of the partially bounded continued fraction $f(a)$ is sufficient for the convergence of $f(a)$. In fact, we have this theorem:

TheOREM 1. Suppose there is a positive integer $k$ such that the sub-

Presented to the Society, December 27, 1955; received by the editors October 27, 1955 and, in revised form, December 27, 1955.

${ }^{1} f(a)$ is called bounded if the sequence $a$ is bounded - a condition equivalent to the boundedness of a certain infinite matrix. Cf. H. S. Wall, Analytic theory of continued fractions, 1948, p. 110. (Referred to later on as AT.)

2 Trans. Amer. Math. Soc. vol. 67 (1949) pp. 368-380. 
sequence $\left\{f_{p}\right\}_{p-k}^{\infty}$ of the sequence of approximants of the partially bounded continued fraction $f(a)$ is bounded. If the even (odd) part of $f(a)$ converges and has the value $v$, then there is an infinite subsequence of the sequence of approximants of the odd (even) part of $f(a)$ which converges to v.

Proof. Let $A_{p}$ and $B_{p}$ be the $p$ th numerator and denominator of $f(a)$, so that $A_{0}=0, A_{1}=1, B_{0}=1, B_{1}=1$,

$$
\begin{aligned}
& A_{p+1}=A_{p}+a_{p} A_{p-1}, \\
& B_{p+1}=B_{p}+a_{p} B_{p-1},
\end{aligned} \quad p=1,2, \cdots,
$$

and

$$
\begin{aligned}
A_{p} B_{p+1}-A_{p+1} B_{p} & =A_{p} B_{p+2}-A_{p+2} B_{p} \\
& =(-1)^{p+1} a_{1} a_{2} \cdots a_{p}, \quad p=1,2, \cdots .
\end{aligned}
$$

Therefore, if $p \geqq k$,

$$
f_{p}-f_{p+1}=\frac{(-1)^{p+1} a_{1} a_{2} \cdots a_{p}}{B_{p} B_{p+1}}, \quad f_{p}-f_{p+2}=\frac{(-1)^{p+1} a_{1} a_{2} \cdots a_{p}}{B_{p} B_{p+2}},
$$

and consequently ${ }^{8}$

$$
-a_{p+2}\left(f_{p}-f_{p+2}\right)\left(f_{p+1}-f_{p+3}\right)=\left(f_{p}-f_{p+1}\right)\left(f_{p+2}-f_{p+3}\right),
$$

or

$$
\left|a_{p+2}\left(f_{p}-f_{p+2}\right)\left(f_{p+1}-f_{p+3}\right)\right|=\left|\left(f_{p}-f_{p+1}\right)\left(f_{p+2}-f_{p+3}\right)\right| .
$$

Suppose the even (odd) part of $f(a)$ converges to $v$ and that no infinite subsequence of the sequence of approximants of the odd (even) part of $f(a)$ converges to $v$. Then, there exists a positive number $c$ and a positive integer $m$ greater than $k$ such that if $p$ is a positive integer greater than $m,\left|\left(f_{p}-f_{p+1}\right)\left(f_{p+2}-f_{p+3}\right)\right| \geqq c$. Let $M$ be a number such that, if $p \geqq k,\left|f_{p}-f_{p+2}\right| \leqq M$, and let $L$ be a number such that, for each positive integer $r$ there exists a positive integer $s$ greater than $r$ for which $\left|a_{s+2}\right| \leqq L$. There exists a positive integer $N$ greater than $m$ such that if $p$ is an integer greater than $N,\left|f_{p}-f_{p+2}\right|$ $<c / M L$ or $\left|f_{p+1}-f_{p+3}\right|<c / M L$. Hence, there exists a positive integer $s$ greater than $N$ such that $c \leqq\left|a_{s+2}\left(f_{8}-f_{8+2}\right)\left(f_{8+1}-f_{8+3}\right)\right| \leqq L M$ $\cdot(c / L M)=c$. This contradiction proves our supposition false and establishes the theorem.

8 This formula expresses the fact that $a_{p+2}$ is a cross-ratio of four successive approximants of $f(a)$. Cf. Wall, Bull. Amer. Math. Soc. vol. 40 (1934) pp. 578-592. 
THEOREM 2. If the even and odd parts of the partially bounded continued fraction $f(a)$ converge, then $f(a)$ converges.

This is an immediate corollary to Theorem 1.

THEOREM 3. There exists a divergent continued fraction $f(a)$ whose even and odd parts converge for which the series $\sum\left|b_{p}\right|$ diverges.

Proof. Let $a$ denote the sequence defined by $a_{p}=(-1)^{p}(p+1)^{2}$, $p=1,2, \cdots$. Since $\left|b_{p} b_{p+1}\right|^{1 / 2}=1 /(p+1) \leqq\left[\left|b_{p}\right|+\left|b_{p+1}\right|\right] / 2$, the series $\sum\left|b_{p}\right|$ diverges. The even part of $f(a)$ is $-f(c) / 3$, where $c$ is the sequence defined by $c_{p}=p^{2}(2 p+1) /(2 p-1), p=1,2, \cdots$, so that the even part of $f(a)$ converges $^{4}$ and its value is a negative number. The odd part of $f(a)$ is $1+2 f(d) / 3$, where $d$ is the sequence defined by $d_{p}=(p+1)^{2}(2 p+1) /(2 p+3), p=1,2, \cdots$, so that the odd part of $f(a)$ converges and its value is a number greater than 1 . Hence $f(a)$ diverges.

A corollary to Theorem 2 is the following theorem of Farinha. Let $E$ denote a bounded closed set in the complex plane which does not contain 0 . If the even and odd parts of $f(a)$ converge uniformly for $a_{i}$ in $E$, then $f(a)$ converges uniformly for $a_{i}$ in $E$.

As an application of Theorem 2, we shall prove the following extension of a theorem of Thron. ${ }^{6}$

THEOREM 4. Suppose $r$ is a positive number not greater than 1 and $s$ $a$ positive number less than $(1+r)^{-2}$. The continued fraction $f(a)$ such that $\left|a_{2 p}\right| \leqq r^{2}$ and $\left|1 / a_{2 p-1}\right| \leqq(1+r)^{-2}-s, p=1,2, \cdots$, is convergent.

Proof. Since $f(a)$ is partially bounded, it suffices to show that its even and odd parts converge.

The even part of $f(a)$ may be written as

$$
\frac{i / a_{1}}{b_{1}+i s}-\frac{c_{1}^{2}}{b_{2}+i s}-\frac{c_{2}^{2}}{b_{3}+i s}-\cdots \text {, }
$$

where

$b_{p}=i+\frac{i}{a_{2 p-1}}+\frac{a_{2 p-2}}{a_{2 p-1}}-i s, \quad c_{p}^{2}=-\frac{a_{2 p}}{a_{2 p+1}}, \quad p=1,2, \cdots,\left(a_{0}=0\right)$.

- AT, pp. 58-59.

S Rev. Fac. Ci. Univ. Coimbra vol. 23 (1954) pp. 17-20.

- Duke Math. J. vol. 10 (1943) pp. 677-685, p. 680. Thron restricts $r$ to be less than 1. Cf. Leighton and Wall, Amer. J. Math. vol. 58 (1936) pp. 267-281, p. 267, for the first theorem of this type. 
For each positive integer $p, \beta_{p}=\operatorname{Im} b_{p} \geqq 2 r(1+r)^{-2}+r^{2} s>0$, and there exists a number $\theta_{p}$ and a number $t_{p}$ such that

$$
\begin{aligned}
\left|c_{p}^{2}\right|=\left(\frac{r}{1+r}\right)^{2} \theta_{p}, \quad \operatorname{Re} c_{p}^{2}= & \left(\frac{r}{1+r}\right)^{2} \theta_{p} t_{p}, \\
& 0 \leqq \theta_{p} \leqq 1,-1 \leqq t_{p} \leqq 1 .
\end{aligned}
$$

Then,

$$
0 \leqq \frac{\left|c_{p}^{2}\right|-\operatorname{Re} c_{p}^{2}}{2 \beta_{p} \beta_{p+1}} \leqq \frac{(1+r)^{2} \theta_{p}\left(1-t_{p}\right)}{2\left(2+r-r \theta_{p} t_{p}\right)\left(2+r-r \theta_{p-1} t_{p-1}\right)} .
$$

Since the derivative with respect to $r$ of the last expression is nonnegative, it does not exceed

$$
\frac{2 \theta_{p}\left(1-t_{p}\right)}{\left(3-\theta_{p} t_{p}\right)\left(3-\theta_{p-1} t_{p-1}\right)}
$$

so that, if $h_{p}=\left(1-t_{p}\right) / 2$ and $g_{p}=\left[1+\theta_{p} h_{p}\right]^{-1}$, we have:

$$
\begin{aligned}
& \left|c_{p}\right|^{2}-\operatorname{Re} c_{p}^{2} \leqq 2 \beta_{p} \beta_{p+1}\left(1-g_{p}\right) g_{p+1}, \\
& 0<g_{p} \leqq 1, p=1,2, \cdots .
\end{aligned}
$$

Thus, the even part of $f(a)$ is positive definite ${ }^{7}$ and, being bounded, is convergent. ${ }^{8}$

The odd part of $f(a)$ may be proved convergent in the same way, completing the proof of Theorem 4.

George Copp 9 has improved Theorem 4 by showing that $f(a)$ converges if there is a positive number $r$ not greater than 1 such that $\left|a_{2 p}\right| \leqq r^{2}$ and $\left|a_{2 p-1}\right| \geqq(1+r)^{2}, p=1,2, \cdots$. He also showed that $f(a)$ converges if there exists a positive number $r$ less than 1 such that, for each positive integer $p,\left|a_{2 p}\right| \leqq r^{2}$ and $\left|a_{2 p-1}\right| \leqq(1-r)^{2}$ or $\left|a_{2 p-1}\right| \geqq(1+r)^{2}$.

UNIVERSITY OF TEXAS

\footnotetext{
AT, p. 69.

8 AT, p. 112.

- George Copp, Some convergence regions for a continued fraction, Dissertation, The University of Texas, 1950.
} 